\title{
Analysis of crystal defects by scanning transmission electron microscopy (STEM) in a modern scanning electron microscope
}

\author{
Cheng Sun ${ }^{*} \mathbb{D}$, Erich Müller, Matthias Meffert and Dagmar Gerthsen
}

\begin{abstract}
Dislocations and stacking faults are important crystal defects, because they strongly influence material properties. As of now, transmission electron microscopy (TEM) is the most frequently used technique to study the properties of single dislocations and stacking faults. Specifically, the Burgers vector $\mathbf{b}$ of dislocations or displacement vector $\mathbf{R}$ of stacking faults can be determined on the basis of the $\mathbf{g} \cdot \mathbf{b}=n(\mathbf{g} \cdot \mathbf{R}=n)$ criterion by setting up different two-beam diffraction conditions with an imaging vector $\mathbf{g}$. Based on the reciprocity theorem, scanning transmission electron microscopy (STEM) can also be applied for defect characterization, but has been less frequently used up to now. In this work, we demonstrate $\mathbf{g} \cdot \mathbf{b}=n(\mathbf{g} \cdot \mathbf{R}=n)$ analyses of dislocations and stacking faults in GaN by STEM imaging in a scanning electron microscope. The instrument is equipped with a STEM detector, double-tilt TEM specimen holder, and a charge-coupled-device camera for the acquisition of on-axis diffraction patterns. The latter two accessories are mandatory to control the specimen orientation, which has not been possible before in a scanning electron microscope.
\end{abstract}

Keywords: Scanning transmission electron microscopy, Scanning electron microscopy, Low electron energy, Transmission electron diffraction, Dislocations, Burgers vector analysis, Stacking faults, GaN

\section{Background}

Dislocations and stacking faults are crystal defects which determine the mechanical properties of materials. They may also strongly influence the electronic properties of semiconductors, because they act as scattering centers for charge carriers or as nonradiative recombination centers in light-emitting devices and solar cells. The characterization of dislocations with respect to dislocation type, dislocation density and distribution is, therefore, of significant interest to understand material properties. Transmission electron microscopy (TEM) has been used for decades to analyze the properties of dislocations and stacking faults. The direction (but not the length) of the Burgers vector $\mathbf{b}$ of dislocations can be determined by exploiting the $\mathbf{g} \cdot \mathbf{b}=n$ criterion $[1,2]$. For this purpose, the sample must be oriented in a two-beam diffraction

*Correspondence: cheng.sun@kit.edu

Laboratorium für Elektronenmikroskopie, Karlsruher Institut für

Technologie (KIT), Engesserstr. 7, 76131 Karlsruhe, Germany condition with an imaging vector $\mathbf{g}$ with only the undiffracted zero-order beam (ZB) and one Bragg reflection strongly excited. Dislocations satisfying $\mathbf{g} \cdot \mathbf{b}=0$ disappear or show only weak residual contrast in bright-field and dark-field TEM images. For two linearly independent imaging vectors $\mathbf{g}_{\mathbf{1}}$ and $\mathbf{g}_{\mathbf{2}}$ satisfying $\mathbf{g} \cdot \mathbf{b}=0$, the Burgers vector can be straightforwardly determined by $\mathbf{b}=\mathbf{g}_{1} \times \mathbf{g}_{2}$. Qualitative considerations and simulations show that double-line contrast is frequently observed in bright-field TEM images for dislocations under $\mathbf{g} \cdot \mathbf{b}=2$ conditions and single-line contrast for $\mathbf{g} \cdot \mathbf{b}=1$ without excitation error $[1,3]$. In more detail, dislocation contrast is also affected by the excitation error $s_{\mathrm{g}}$ from the Bragg reflecting condition and depends on the dislocation type, TEM sample thickness, dislocation depth in the sample, and degree of crystal anisotropy which may lead to deviations from the contrast behavior described above [1, 4].

Scanning transmission electron microscopy (STEM) has been less frequently considered for defect characterization, although the reciprocity theorem suggests 
equivalent diffraction contrast for STEM and TEM imaging [5]. After some early work on defect imaging by bright-field (BF)-STEM [6-8], Philipps et al. [9], Su et al. [10], and Zhu et al. [11] more recently investigated dislocations and stacking faults by STEM and demonstrated Burgers vector determination by experiments and simulations. They also pointed out that the application of STEM provides advantages compared to TEM, because STEM can be performed on thicker TEM samples. Moreover, bend contours and thickness fringes are less pronounced due to the convergent probe, while defect contrast is maintained if BF-STEM collection angles are moderately increased.

STEM can be also carried out in a scanning electron microscope (SEM) if the instrument is equipped with a STEM detector. However, defect analyses were hampered up to now, because the specimen orientation could not be precisely controlled due to the lack of cameras to acquire on-axis transmission electron diffraction (TED) patterns and double-tilt TEM specimen holders that are necessary to orient specimens in a two-beam condition. These accessories have become available only recently which explains the lack of defect investigations by STEM in a scanning electron microscope which will be denoted as low-keV STEM in the following. Preliminary investigations of dislocation Burgers vectors in $\mathrm{InN}$ by low-keV STEM were presented by us [12]. In addition, recently, Callahan et al. [13] presented defect analyses by low-keV STEM in samples with a priori known orientation by comparing experimental images and simulations in various materials, but they were not able to systematically exploit the $\mathbf{g} \cdot \mathbf{b}=n$ criterion for instrumental reasons.

In this work, we present systematic analyses of dislocation Burgers vectors and displacement vectors of stacking faults performed by low-keV STEM in a scanning electron microscope. The analyses were carried out in a specially configured instrument which allows to control the specimen orientation and set up defined two-beam conditions by taking TED patterns. We used GaN as a test material, because the properties of dislocations and stacking faults are well known in this material. It will be also demonstrated that the maximum electron energy of $30 \mathrm{keV}$ well suffices to study defects in FIB-prepared TEM specimens and dislocation contrast is still recognizable at even lower electron energies such as $10 \mathrm{keV}$.

\section{Methods}

Dislocations and stacking faults in an epitaxial (0001) $\mathrm{GaN}$ layer grown on a (0001) $\mathrm{Al}_{2} \mathrm{O}_{3}$ substrate were analyzed. Electron-transparent cross-sectional specimens with either $[10 \overline{1} 0]$ or $[11 \overline{2} 0]$ zone-axis orientation were prepared by FIB milling in a Helios Nanolab G4 FX dualbeam instrument (Thermo Fisher) using the lift-out technique [14]. The surface of the GaN layer was covered by $\mathrm{Pt}$ to protect the specimen from damage by $\mathrm{Ga}$-ion milling during lamella preparation. The thickness of the FIB-prepared specimens is estimated to be between 50 and $100 \mathrm{~nm}$. No particular effort was undertaken to prepare extremely thin specimens.

Low-keV STEM and SEM imaging was performed with the combined FIB/SEM-instrument Helios Nanolab G4 FX (cf. Scheme in Fig. 1a). The instrument is equipped with a double-tilt specimen holder for electron-transparent samples. Large $\alpha$ and $\beta$ tilt angle ranges of $-10^{\circ}$ to $170^{\circ}$ and $-190^{\circ}$ to $10^{\circ}$, respectively, are favorable for defect characterization. A retractable e-Flash ${ }^{\mathrm{HR}}$ chargecoupled-device (CCD) camera (Bruker OPTIMUS ${ }^{\mathrm{TM}}$ ) is mounted below the specimen for acquiring TED patterns. The following procedure is used to orient a specimen in a two-beam condition. First, the $\alpha$ and $\beta$ tilt angles for a certain two-beam condition are set up by acquiring TED patterns with the CCD camera inserted. Before STEM images can be taken, the CCD camera and sample holder have to be retracted and the STEM detector is inserted. With the STEM detector in place, the sample holder is inserted again with the $\alpha$ and $\beta$ tilt angle previously determined which may lead to small deviations from the selected two-beam condition (estimated error $\pm 0.1^{\circ}$ ).

Defect characterization is performed by BF-STEM using the BF segment of the multi-segmented semiconductor STEM detector. The beam convergence (half) angle of $1.7 \mathrm{mrad}$ in the field-free imaging mode is measured by the technique suggested by Lyman et al. [15]. We cannot control the convergence angle in the Helios Nanolab G4 FX, because it is fixed by the manufacturer setup. The lens system and aperture diameters are prealigned in the field-free mode to achieve small convergence angles for beam currents between $1.6 \mathrm{pA}$ and $0.8 \mathrm{nA}$. To illustrate the influence of the convergence angle on BF-STEM contrast, we used the immersion mode with a convergence angle of $10 \mathrm{mrad}$. However, the immersion mode is in general not well suited for diffraction contrast analyses, because TED patterns are distorted and overlap of Bragg disks may occur. The convergence angle in the field-free mode is significantly smaller than the $7 \mathrm{mrad}$ collection (half) angle of the BFSTEM detector segment. The latter can essentially not be changed, because a projection lens system is missing and only a small variation of $7 \%$ is possible by changing the working distance.

All relevant Bragg angles of GaN reflections are larger than $12 \mathrm{mrad}$ at $30 \mathrm{keV}$. The only exception is the (0001) reflection which can be dynamically excited in the $[11 \overline{2} 0]$ zone axis. Large Bragg angles compared to convergence and collection angles are advantageous, because overlap 


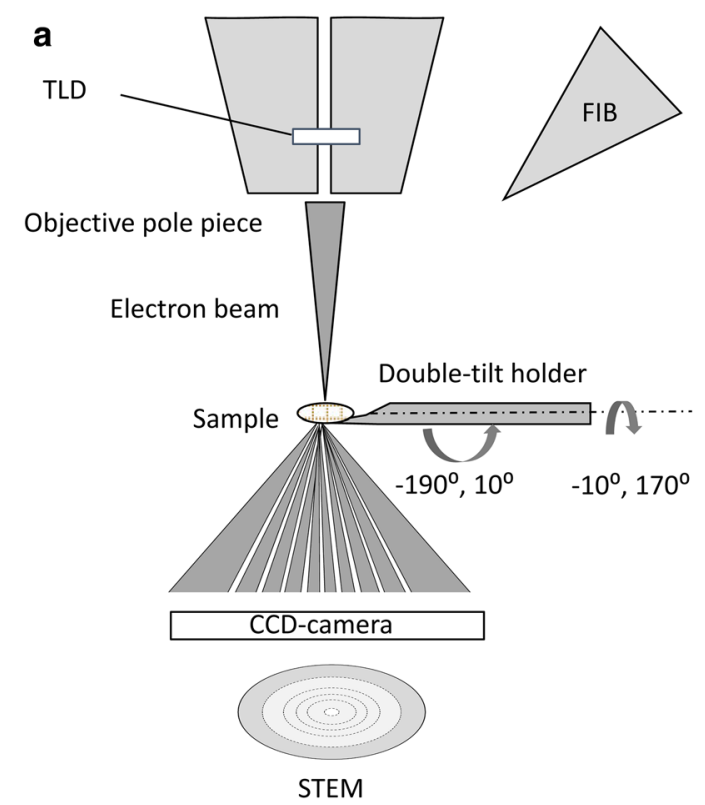

\section{b}

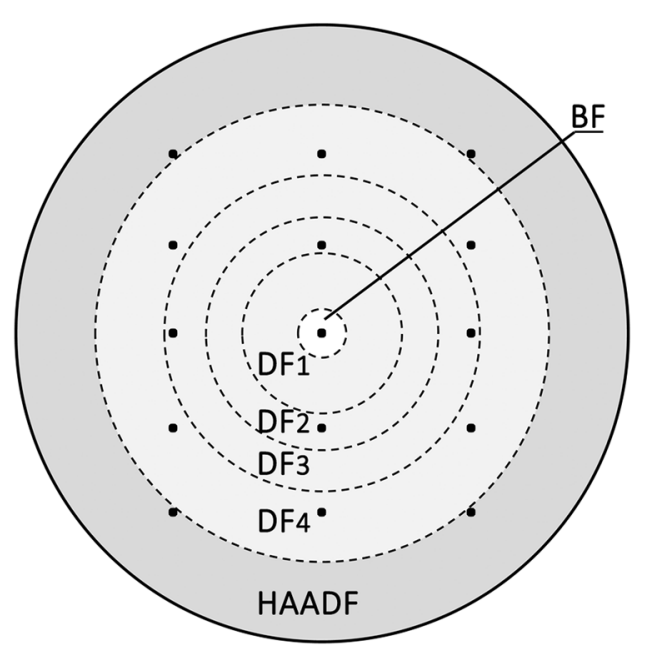

Fig. 1 a Scheme of Helios Nanolab G4 FX dual-beam instrument with double-tilt holder for TEM specimens, STEM detector, through-the-lens detector (TLD), retractable on-axis CCD camera, and FIB column. b Top view of multi-segmented STEM detector with bright-field (BF), four narrow annular dark field (ADF), and high-angle annular dark-field (HAADF) segments. The positions of Bragg reflections for GaN in [1010] zone axis at $30 \mathrm{keV}$ are indicated

of diffraction disks does not occur and distinct separation of ZB and Bragg reflections on the BF-STEM detector is achieved. This is illustrated in Fig. 1b which shows a scheme of the multi-segmented STEM detector superimposed by the TED pattern of GaN in $[10 \overline{1} 0]$ zone-axis orientation. Bragg disks and STEM detector segments, except for the large high-angle annular dark-field segment, are drawn to scale in Fig. 1b. Dark-field (DF) STEM images with $\mathbf{g}_{(\mathbf{0 0 0 2})}$ were additionally acquired using the DF2 segment of the STEM detector which is illuminated solely by the (0002) reflection.

A through-the-lens detector (TLD) is used for secondary-electron SEM imaging yielding topography contrast of the specimen surface regions analyzed by STEM. Topography imaging is applied to assess sample thickness variations and resulting BF-STEM contrast changes.

Figure 2 shows a spherical Kikuchi map of GaN simulated for $30 \mathrm{keV}$ assuming kinematical diffraction conditions. The simulation was performed with the Esprit 2.1 software (Bruker). The Kikuchi map shows the [1010] and $[11 \overline{2} 0]$ zone axes and the three two-beam conditions used in this work. Two-beam conditions were set up by tilting along Kikuchi lines, whose visibility depends on the local sample thickness. The visibility of Kikuchi lines can be improved by increasing the exposure time and electron-beam current, which may eventually lead to local contamination of the sample. Large-angle tilts are required to tilt from $\mathbf{g}_{(\mathbf{1 - 2 1 0})}$ to $\mathbf{g}_{(\mathbf{1 - 1 0 0}) \text {. We note that }}$

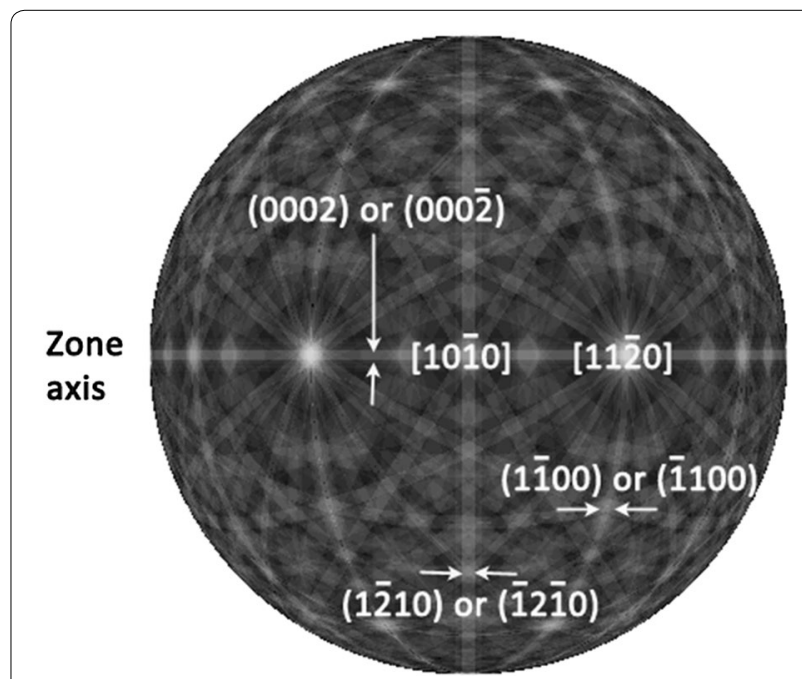

Fig. 2 Spherical Kikuchi map of GaN. The arrows indicate two-beam conditions that were used for Burgers vector determination in this work

two-beam conditions at $30 \mathrm{keV}$ can be realized with less pronounced excitation of other Bragg reflections than at higher electron energies due to the stronger curvature of the Ewald sphere. 


\section{Results and discussion}

Figure 3 shows dislocations in $30 \mathrm{keV}$ cross section BFSTEM images in an epitaxial GaN layer. A high density of threading dislocations is typically observed in epitaxial $\mathrm{GaN}$ layers grown on $\mathrm{Al}_{2} \mathrm{O}_{3}(0001)$. The dislocations are generated during $\mathrm{GaN}$ deposition on the $\mathrm{Al}_{2} \mathrm{O}_{3}$ substrate as a consequence of the lattice parameter mismatch and different thermal expansion coefficients of $\mathrm{GaN}$ and $\mathrm{Al}_{2} \mathrm{O}_{3}[16]$.

The same specimen region is imaged under three different two-beam conditions (Fig. 3a-c) using $\mathbf{g}_{(000-2)}$, $\mathbf{g}_{(\mathbf{1 - 2 1 0})}$, and $\mathbf{g}_{(\mathbf{1 - 1 0 0})}$ as demonstrated by the TED patterns (Fig. 3d-f). Threading dislocations appear as dark lines, which are typically oriented along or close to the [0001] growth direction. Seven dislocations are marked in the images which show different contrasts in Fig. 3a-c. Dislocations 1-6 show strong contrast in Fig. 3a using $\mathbf{g}_{(000-2)}$ predominantly with double lines.
Dislocation 7 appears with weak residual contrast and is considered to be out of contrast according to $\mathbf{g} \cdot \mathbf{b}=0$ in Fig. 3a. The latter dislocation shows strong contrast in Fig. 3b taken with $\mathbf{g}_{(\mathbf{1 - 2 1 0})}$, while dislocation 1 is out of contrast here. Double-line contrast is observed for most other dislocations. Dislocations 1 and 7 are invisible using $\mathbf{g}_{(\mathbf{1 - 1 0 0 )}}$ (Fig. 3c) and all the other dislocations show single-line contrast. The result of the contrast analyses for dislocations 1-7 is summarized in Table 1 , where ' \pm ' symbols indicate visibility or extinction of dislocations contrast.

Only dislocations 1 and 7 show contrast extinction for two different imaging vectors which allow straightforward Burgers vector determination. According to the (in)visibility of these dislocations, the Burgers vectors must be parallel to [0001] (dislocation 1) and parallel to $[11 \overline{2} 0]$ (dislocation 7 ). With dislocation line directions along or close to [0001], dislocation 1 is a screw
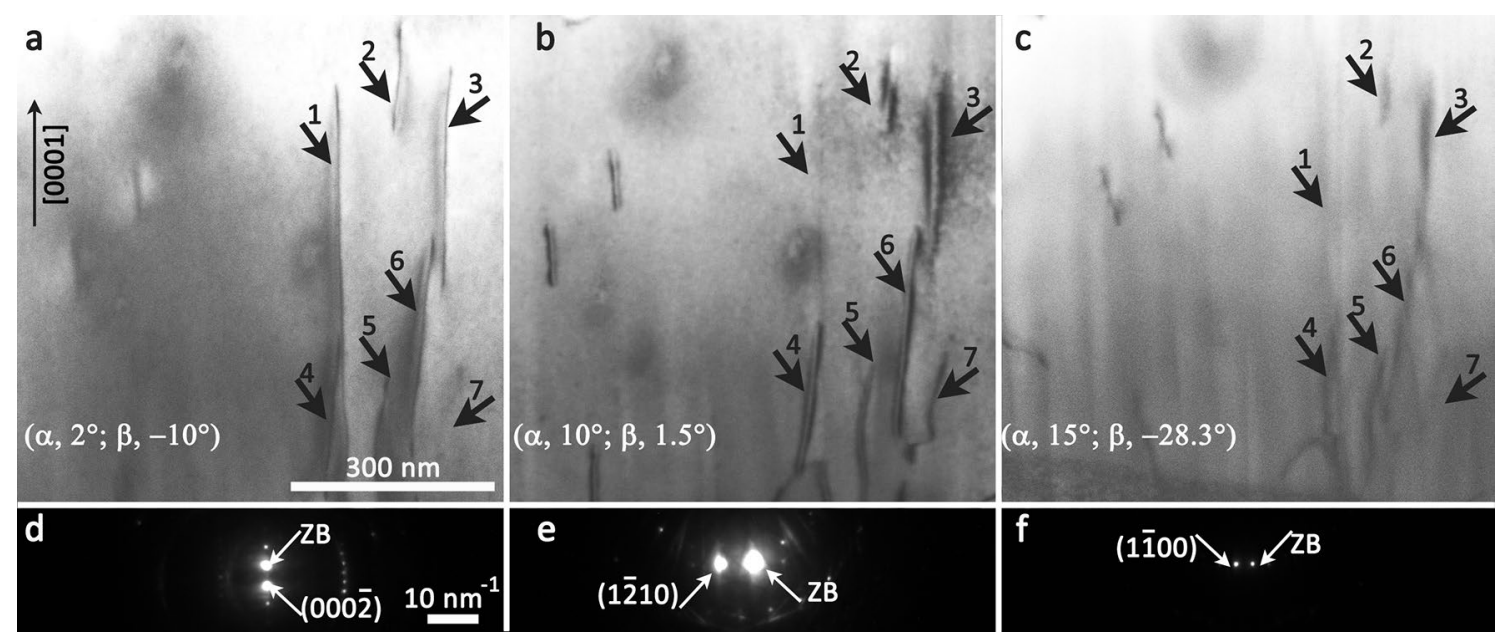

Fig. $330 \mathrm{keV}$ cross-sectional BF-STEM images of the epitaxial GaN layer and corresponding TED pattern for different two-beam conditions $\mathbf{a}$, $\mathbf{d}$ $\mathbf{g}_{(\mathbf{0 0 0 - 2})} \mathbf{b}, \mathbf{e} \mathbf{g}_{(\mathbf{1 - 2 1 0})}$, and $\mathbf{c}, \mathbf{f}_{(\mathbf{1 - 1 0 0 )}}$. ZB and strongly excited Bragg reflections are marked in $\mathbf{d}$-f. The settings of the $a$ - and $\beta$-tilt angle are given in the BF-STEM images. Scale bars in $\mathbf{a}$ and $\mathbf{d}$ apply to all BF-STEM images and diffraction patterns, respectively. Dark spot-like regions result from contamination that occurred during sample orientation by acquiring diffraction patterns with a high beam current

Table 1 Visibility (+)/extinction (-) of dislocation contrast and $\mathbf{g} \cdot \mathbf{b}$ of dislocations 1-7 in Fig. 3 for different $\mathbf{g}$

\begin{tabular}{|c|c|c|c|c|c|c|c|}
\hline Two-beam condition & 1 & 2 & 3 & 4 & 5 & 6 & 7 \\
\hline $\begin{array}{l}(000-2) \\
|\mathbf{g} \cdot \mathbf{b}|\end{array}$ & $\begin{array}{l}+ \\
2\end{array}$ & $\begin{array}{l}+ \\
2\end{array}$ & $\begin{array}{l}+ \\
2\end{array}$ & $\begin{array}{l}+ \\
2\end{array}$ & $\begin{array}{l}+ \\
2\end{array}$ & $\begin{array}{l}+ \\
2\end{array}$ & $\overline{0}$ \\
\hline $\begin{array}{l}(1-210) \\
|\mathbf{g} \cdot \mathbf{b}|\end{array}$ & $\frac{-}{0}$ & $\begin{array}{l}+ \\
2\end{array}$ & $\begin{array}{l}+ \\
2\end{array}$ & $\begin{array}{l}+ \\
2\end{array}$ & $\begin{array}{l}+ \\
2\end{array}$ & $\begin{array}{l}+ \\
2\end{array}$ & $\begin{array}{c}+ \\
1\end{array}$ \\
\hline $\begin{array}{l}(1-100) \\
|\mathbf{g} \cdot \mathbf{b}|\end{array}$ & $\frac{-}{0}$ & $\begin{array}{c}+ \\
1\end{array}$ & $\begin{array}{c}+ \\
1\end{array}$ & $\begin{array}{c}+ \\
1\end{array}$ & $\begin{array}{c}+ \\
1\end{array}$ & $\begin{array}{c}+ \\
1\end{array}$ & $\frac{-}{0}$ \\
\hline Burgers vector & {$[0001]$} & $\begin{array}{l}1 / 3[1 \overline{2} 13] \\
1 / 3[1 \overline{2} 1 \overline{3}]\end{array}$ & $\begin{array}{l}1 / 3[1 \overline{2} 13] \\
1 / 3[1 \overline{2} 1 \overline{3}]\end{array}$ & $\begin{array}{l}1 / 3[1 \overline{2} 13] \\
1 / 3[1 \overline{2} 1 \overline{3}]\end{array}$ & $\begin{array}{l}1 / 3[1 \overline{2} 13] \\
1 / 3[1 \overline{2} 1 \overline{3}]\end{array}$ & $\begin{array}{l}1 / 3[1 \overline{2} 13] \\
1 / 3[1 \overline{2} 1 \overline{3}]\end{array}$ & $1 / 3[11 \overline{2} 0]$ \\
\hline Dislocation type & Screw & Mixed & Mixed & Mixed & Mixed & Mixed & Edge \\
\hline
\end{tabular}


and dislocation 7 an edge dislocation. The other dislocations in Fig. 3 do not show any contrast extinctions. However, even without contrast extinctions, we can assign Burgers vectors based on the previous work on dislocations in hexagonal lattices in general and specifically in GaN [17-19]. In general, Burgers vectors of the type [0001] (corresponding to the direction and length of the c-lattice parameter), $1 / 3<11 \overline{2} 0>$ (corresponding to the direction and length of the a-lattice parameter), and $1 / 3<11 \overline{2} 3>$ (corresponding to the direction and length of $\mathbf{c}+\mathbf{a})$ were previously observed. The acute brackets indicate that several crystallographic equivalent directions of this type exist, e.g., there are six independent Burgers vectors for $1 / 3<11 \overline{2} 3>$ dislocations. Due to the pre-knowledge of possible Burgers vectors, further assignments can be made. From the visibility of dislocations 2-6 for $\mathbf{g}_{(\mathbf{0 0 0 - 2})}, \mathbf{g}_{(\mathbf{1 - 2 1 0})}$, and $\mathbf{g}_{(1-100)}$, we can conclude that they must be mixed dislocations. However, only four out of six possible Burgers vectors $(1 / 3[\overline{2} 113], 1 / 3[\overline{2} 11 \overline{3}], 1 / 3[1 \overline{2} 13], 1 / 3[1 \overline{2} 1 \overline{3}])$ for mixed dislocations are expected, because dislocations with $1 / 3[11 \overline{2} 3]$ and $1 / 3[11 \overline{23}]$ should be out of contrast for $\mathbf{g}_{(\mathbf{1 - 1 0 0 )} \text {. }}$

Although caution is necessary to interpret details of dislocation line contrast, different appearances of dislocation line contrast in Fig. 3a-c can be exploited for further Burgers vector specification. The observation of double-line contrast for most dislocations in Fig. 3a, b suggests $|\mathbf{g} \cdot \mathbf{b}|=2$ (for $s_{\mathrm{g}}=0$ ), because double-line contrast is frequently obtained under these conditions $[1,3]$. The mixed dislocations with pronounced double-line contrast in Fig. 3b are, therefore, only compatible with $1 / 3[1 \overline{2} 13]$ and $1 / 3[1 \overline{2} 1 \overline{3}]$ Burgers vectors to fullfill $|\mathbf{g} \cdot \mathbf{b}|=2$ using $\mathbf{g}_{(\mathbf{1 - 2 1 0})}$. This assignment is consistent with Fig. 3c, where these dislocations show single-line contrast as expected for $|\mathbf{g} \cdot \mathbf{b}|=1$. Double-line contrast of dislocations in $\mathrm{GaN}$ under $|\mathbf{g} \cdot \mathbf{b}|=2$ conditions is also seen in a TEM image published by Ponce et al. [17], although the authors did not exploit this contrast feature in their Burgers vector analyses.

We exclude that double-line contrast results from dislocation dissociation into partial dislocations, although high-resolution annular dark-field STEM performed by Hirsch et al. [20] indicates dissociation of threading dislocation cores with $1 / 3<11 \overline{2} 3>$ Burgers vector in an epitaxial GaN layer. However, dissociation widths are only in the order of nanometers, which is far too small to be resolved by BF-STEM imaging with $s_{\mathrm{g}} \approx 0$. In addition to dislocations, broad dark line-like contrast features along the [0001] direction are visible in Fig. 3c. These features can be assigned to thickness variations of the FIB-prepared TEM lamella, which can be deduced from topography contrast of secondary-electron SEM images of the same specimen region (cf. Additional file 1: Fig. S1).

The images in Fig. 3 were taken without excitation error, because we aimed at exploiting double-line contrast for $|\mathbf{g} \cdot \mathbf{b}|=2$ which is only observed for $s_{\mathrm{g}}=0$. However, the sharpness of dislocation line contrast can be improved if two-beam conditions with an excitation error are set up. The influence of $s_{\mathrm{g}}$ is illustrated in Fig. 4 that presents $\mathbf{g}_{(\mathbf{0 0 0 2})}$ BF-STEM images taken without excitation error (Fig. 4a) and with $s_{\mathrm{g}}=0.054 \mathrm{~nm}^{-1}$ (Fig. 4b). In the first case, the contrast of the dislocation line is diffuse, whereas sharp dark dislocation lines are observed in Fig. 4b. In addition, two-beam DF-STEM imaging is possible, because the (0002) reflection is located on the DF2-segment of the STEM detector (cf. Fig. 1b). Comparing DF-STEM images acquired without excitation error (Fig. 4c) and under (g, 3g) weakbeam conditions (Fig. 4d) clearly reveals the increase of sharpness of dislocation line contrast under weak-beam conditions. The sharpness enhancement of dislocation contrast can be attributed to the correlation of the width of dislocation contrast with extinction distance $\xi_{\mathrm{g}}[21]$ and the reduction of $\xi_{\mathrm{g}}$ with increasing $s_{\mathrm{g}}$.

Figure 3 demonstrates that dislocations show distinct Bragg diffraction contrast in $30 \mathrm{keV}$ STEM images that can be exploited for Burgers vector determination. Figure 5 illustrates that Bragg diffraction contrast of dislocation is visible at even lower electron energies. Using $\mathbf{g}_{(-1100)}$ or $\mathbf{g}_{(\mathbf{1 - 1 0 0 )})}$ the same specimen region is imaged at $30 \mathrm{keV}$ (Fig. 5a, d) and reduced electron energies of $20 \mathrm{keV}$ (Fig. 5b, e) and $10 \mathrm{keV}$ (Fig. 5c, f). The reduction of the electron energy is obvious from the increasing distance between $\mathrm{ZB}$ and excited Bragg reflection (Fig. $5 \mathrm{~d}-\mathrm{f}$ ). Due to the weak dislocation contrast at $10 \mathrm{keV}$ (Fig. 5c) a higher electron current was used $(1.6 \mathrm{nA}$ as opposed to $50 \mathrm{pA}$ at 20 and $30 \mathrm{keV}$ ) which leads to loss of detail in dislocation contrast. An estimate of the local specimen thickness can be obtained from contrast oscillations of the dislocation marked by a black arrow in Fig. 5a, which traverses the specimen at an inclined direction. A bright-dark intensity oscillation along the dislocation line corresponds to a change of specimen thickness given by $\xi_{\mathrm{g}}$ which is $36 \mathrm{~nm}$ at $30 \mathrm{keV}$ for $\mathbf{g}_{(-1100)}$ and $s_{\mathrm{g}}=0$. For the dislocation marked by the arrow in Fig. $5 \mathrm{a}$, the local sample thickness is estimated to be $90 \pm 18 \mathrm{~nm}$ corresponding to 2.5 $\xi_{\mathrm{g}}$. This demonstrates that the specimen is not exceptionally thin, but corresponds to typical thicknesses of FIB-milled TEM specimens. We note that Fig. 5a also shows pronounced bend contours associated with the change of the orientation of the crystal lattice with respect to the incident electron beam. It is possible to reduce bend contours by enlarging the convergence 


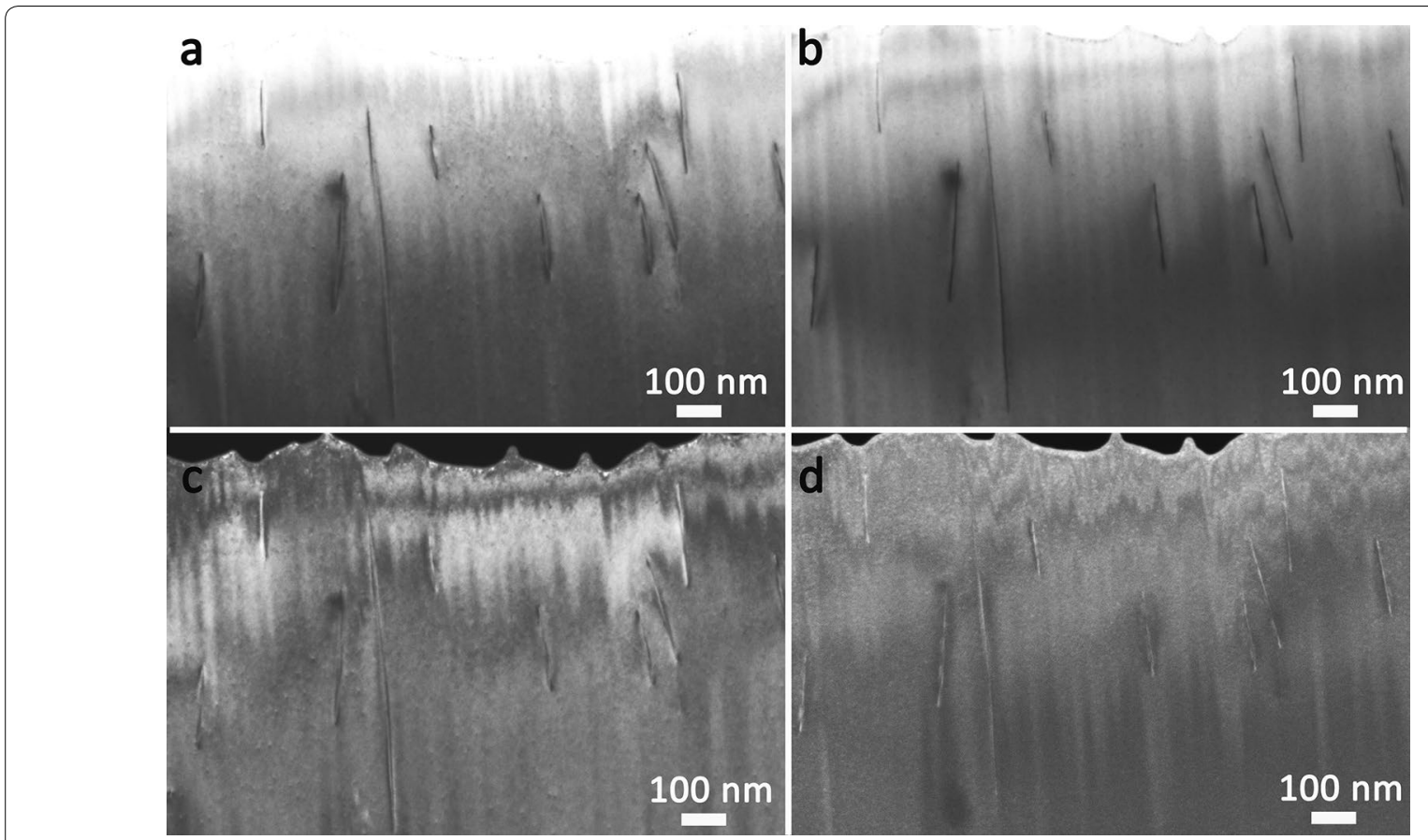

Fig. 430 keV STEM cross-section images of the same region in GaN taken under different conditions $\mathbf{a}$ BF-STEM image with $\mathbf{g}_{(\mathbf{0 0 0 2})}$ and $s_{\mathrm{g}}=0$, $\mathbf{b}$ BF-STEM image with $\mathbf{g}_{(\mathbf{0 0 0 2})}$ and $\mathrm{s}_{\mathrm{g}}=0.054 \mathrm{~nm}^{-1}$, c DF-STEM image with $\mathbf{g}_{(\mathbf{0 0 0 2})}$ and $\mathrm{s}_{\mathrm{g}}=0$ and $\mathbf{d}(\mathbf{g}, 3 \mathbf{g})$ weak-beam DF-STEM
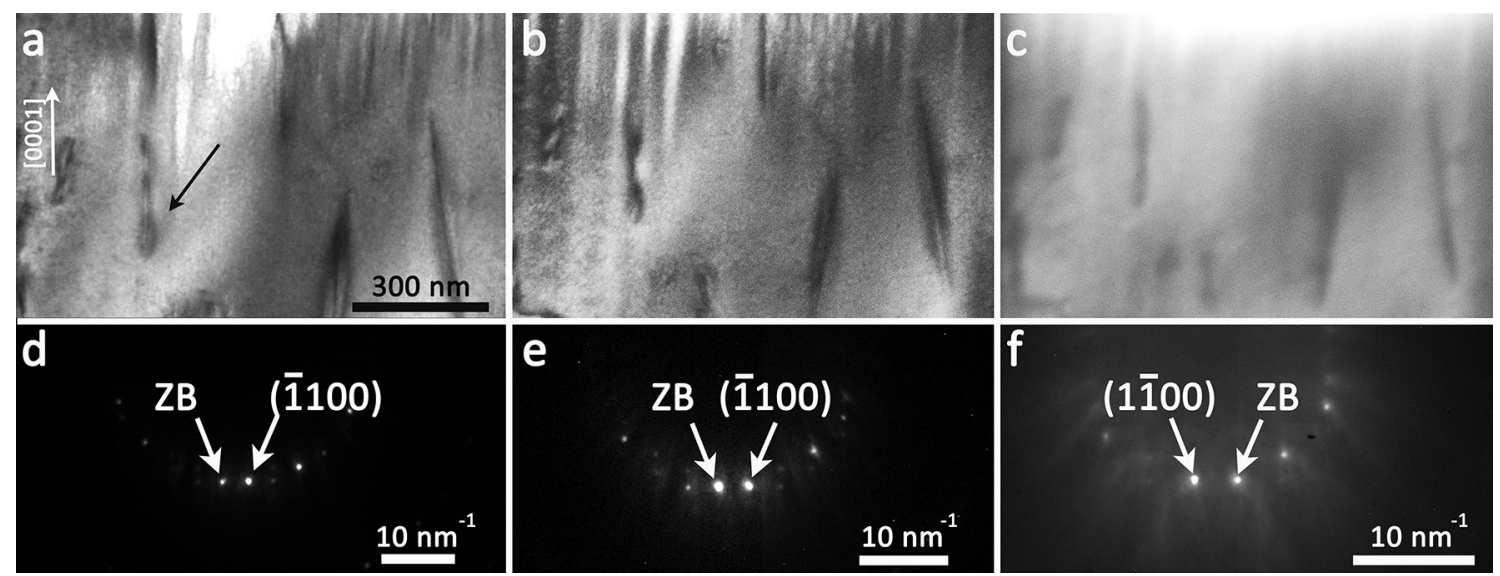

Fig. 5 BF-STEM images of the same specimen region in GaN and corresponding TED pattern for $\mathbf{g}_{(1-100)} / \mathbf{g}_{(-1100)}$ taken at a, d $30 \mathrm{keV}$, $\mathbf{b}, \mathbf{e} 20 \mathrm{keV}$, and $\mathbf{c}, \mathbf{f} 10 \mathrm{keV}$. ZB and strongly excited Bragg reflections are marked in $\mathbf{d}-\mathbf{f}$. Scale bar in $\mathbf{a}$ applies to all BF-STEM images

angle which is shown in Additional file 1: Fig. S3 using the immersion-imaging mode.

Stacking faults are another common defect type in GaN. They are characterized by a displacement vector $\mathbf{R}$ which describes the shift of the stacking fault plane with respect to its position in the undisturbed lattice. Stacking faults can be formed by the dissociation of complete dislocations into two partial dislocations with a stacking fault plane in between. Alternatively, they can be generated during material fabrication if a plane is displaced from its position with respect to the undisturbed lattice. Stacking faults can be investigated by BF-STEM in analogy to TEM, where $\mathbf{g} \cdot \mathbf{R}=0$ leads to contrast extinction of the stacking fault. In addition to this extinction condition, invisibility of $\mathbf{g} \cdot \mathbf{R}=n$ ( $n$ : integer number) occurs, because in these cases, the vector $\mathbf{R}$ moves the reflecting planes 
normal to themselves by a distance equal to an integer number of the spacing between the planes. Several stacking fault types were previously identified in $\mathrm{GaN}$ which differ in the stacking order of the (0001) basal planes [19, 22]. The stacking order of the (0001) planes in the undisturbed wurtzite lattice is ...aAbBaAbB...., where capital and small letters indicate the stacking positions of the $\mathrm{Ga}$ and $\mathrm{N}$ planes, respectively. Two intrinsic stacking faults were found with stacking orders ... $\mathrm{aAbBaAbB} \mid \mathrm{cCbBcC} \ldots$ ( $I_{1}$-type stacking fault) or ...aAbBaAbB $\mid \mathrm{cCaAcCaAcC} \ldots$ ( $I_{2}$-type stacking fault). Furthermore, an extrinsic stacking fault with ...aAbBaAbB $|\mathrm{cC}| \mathrm{aAbBaAbB} .$. (E-type stacking fault) stacking order was observed. The faults are characterized by displacement vectors $R=1 / 6<20 \overline{2} 3>$ $\left(I_{1}\right), R=1 / 3<10 \overline{1} 0>\left(I_{2}\right)$, and $R=1 / 2[0001](E)$.

The $30 \mathrm{keV}$ cross-sectional BF-STEM image of the GaN layer Fig. 6a shows several stacking faults on the (0001) basal plane in the region marked by the back arrow. These stacking faults are out of contrast for $\mathbf{g}_{(\mathbf{0 0 0 2})}$ and $\mathbf{g}_{(\mathbf{1 - 2 1 0})}$ (Fig. 6b, c). The (in)visibility of the stacking faults is compatible with $I_{1^{-}}$or $I_{2}$-type stacking faults, while extrinsic stacking faults can be ruled out on the basis of the $\mathbf{g} \cdot \mathbf{R}=n$ criterion. Distinction between $I_{1}$ and $I_{2}$-type intrinsic stacking faults is not possible with the three applied $\mathbf{g}$ and requires imaging with additional g vectors. In addition, thickness fringes are observed in Fig. $6 \mathrm{~b}, \mathrm{c}$ which also yield an estimate of the specimen thickness at the location of the stacking faults. Figure $6 \mathrm{~b}$ shows a dark fringe at the specimen edge indicating that the local thickness must be at least $0.5 \xi_{\mathrm{g}}$. The majority of the stacking faults are located in a region with a thickness of at least $1.5 \xi_{\mathrm{g}}$ which corresponds to a thickness of $37.5 \mathrm{~nm}$ with $\xi_{\mathbf{g}(\mathbf{0 0 0 2})}=25 \mathrm{~nm}$. This consideration demonstrates again that Bragg diffraction contrast is not impaired at $30 \mathrm{keV}$ at moderate specimen thickness.

We have shown in this work that diffraction contrast analyses of defects can be well performed by low-keV STEM in a scanning electron microscope. There are advantages and drawbacks connected with diffraction contrast imaging by low-keV and high-keV techniques which are discussed in the following.

Advantages of low-keV STEM in scanning electron microscopes are:

- SEM detectors are available which allow correlative SEM imaging of surface topography and STEM imaging from the same specimen region. Information on specimen topography can be helpful for interpretation of STEM contrast (see Figs. S1 and S2 in Additional file 1 which show surface topography images of the TEM lamella). Combination with other imaging techniques in scanning electron microscopes (cathodoluminescence, electron-beam-induced current, etc.) is possible which are not available in transmission electron microscopes (for a more extensive discussion, refer to Callahan et al. [13]).

- Low-keV STEM is advantageous for samples which are sensitive towards knock-on damage.

- In a combined FIB/SEM instrument, materials sensitive towards air exposure can be prepared and subsequently investigated by low-keV STEM without removing them from the microscope.

- The curvature of the Ewald sphere is stronger at low electron energies which allows to set up two-beam conditions with weaker excitation of other Bragg reflections.
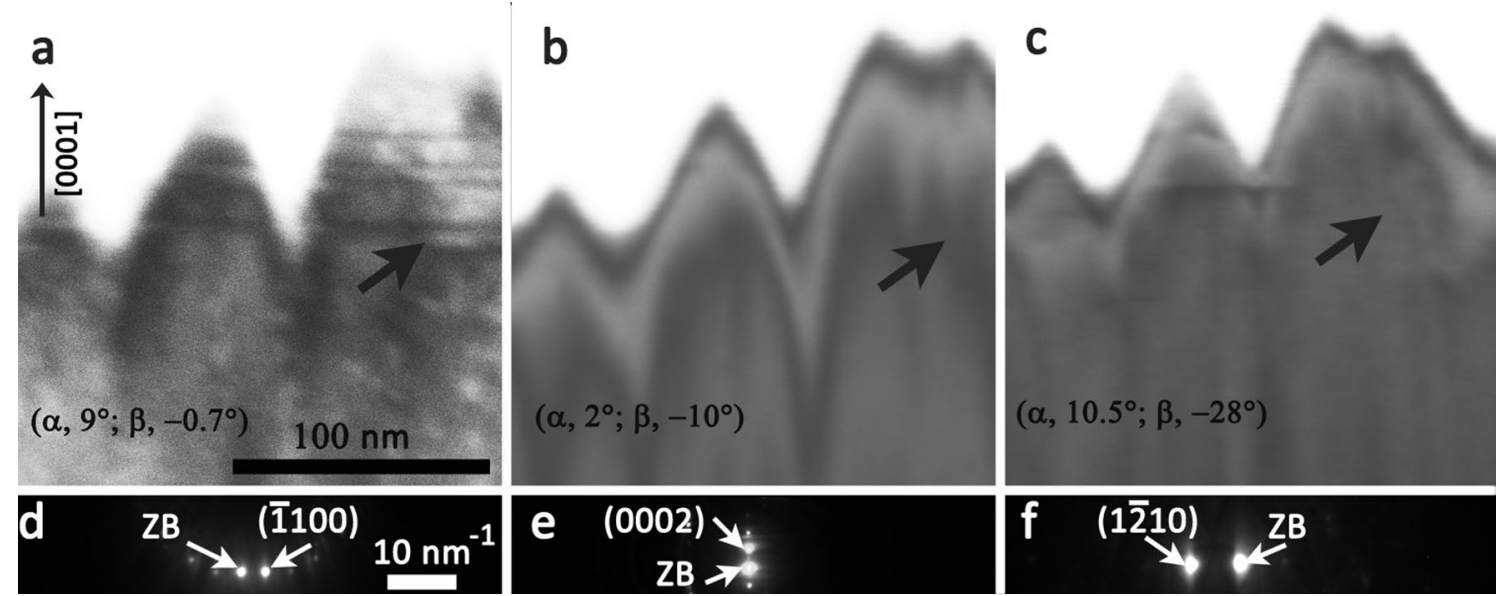

Fig. 630 keV BF-STEM cross-section images of the epitaxial GaN layer and corresponding TED pattern for different two-beam conditions using (a, d) $\mathbf{g}_{(-1100)},(\mathbf{b}, \mathbf{e}) \mathbf{g}_{(\mathbf{0 0 0 2})},(\mathbf{c}, \mathbf{f}) \mathbf{g}_{(\mathbf{1 - 2 1 0})}$. Scale bars in $\mathbf{a}$ and $\mathbf{d}$ apply to all BF-STEM images and diffraction patterns, respectively 
- Scanning electron microscopes are in general more widely available than transmission electron microscopes, but the necessary accessories have to be installed.

Disadvantages of low-keV STEM are:

- Although all mandatory accessories for the control of the specimen orientation are, meanwhile, commercially available (STEM detector, on-axis CCD camera for TED patterns, and double-tilt specimen holder), the choice of convergence and collection angles is limited. This applies in particular to the collection angle, because a projection lens system is not available in scanning electron microscopes. This prevents contrast optimization (reduction of bend or thickness contours) by choosing, e.g., the same convergence and collection angles as outlined by Zhu et al. [11].

- The specimen thickness must be smaller than for high-energy techniques, in particular compared to high-energy STEM.

- Low-keV STEM is not suited for electrically insulating materials which suffer from damage by radiolysis.

- A practical disadvantage in our present experimental setup is that changing between imaging and diffraction mode requires the retraction of the specimen holder and insertion/removal of the CCD camera and STEM detector. This reduces the precision at which two-beam conditions and excitation errors can be set up.

With respect to tolerable maximum specimen thicknesses, a systematic study would be interesting, where specimen thicknesses for diffraction contrast imaging of defects by low-energy STEM, high-energy STEM, and TEM are compared. Another interesting point is the width of dislocation contrast which is correlated with $\xi_{\mathrm{g}}$. This suggests that the reduction of extinction lengths at low electron energies could lead to narrower dislocation contrast and better resolution of dislocation lines at high dislocation densities.

\section{Conclusions}

Exploiting the $\mathbf{g} \cdot \mathbf{R}=n$ and $\mathbf{g} \cdot \mathbf{b}=n$ criterion, respectively, we have shown in this work that dislocation Burgers vectors $\mathbf{b}$ and displacement vectors $\mathbf{R}$ of stacking faults in $\mathrm{GaN}$ can be determined by low-keV STEM in a scanning electron microscope. Using FIB-prepared TEM specimens, which were not specifically optimized for a particularly small sample thickness, diffraction contrast of dislocations and stacking faults is not impaired at $30 \mathrm{keV}$ and even lower electron energies such as $20 \mathrm{eV}$ and $10 \mathrm{keV}$ can be used. In addition to the invisibility of defect contrast, specific dislocation contrast features such as single-line and double-line contrasts appear and can be exploited in Burgers vector analyses. This demonstrates that diffraction contrast analyses of defects, that are traditionally performed in a transmission electron microscope, can be well carried out by low-keV STEM in a modern scanning electron microscope equipped with STEM detector, on-axis CCD camera, and a double-tilt specimen holder. Due to the full control of the specimen orientation, additional information from TEM experiments or image simulations is not necessary. Moreover, the surface topography of the prepared TEM sample can be imaged by secondary-electron SEM which supports STEM contrast interpretation by revealing specimen thickness changes and other topography effects that influences STEM contrast. Overall, the capabilities of scanning electron microscopes are greatly enhanced and diffraction contrast analyses can be performed that were previously only possible in transmission electron microscopes at high electron energies. Further interesting studies comprise systematic investigations of maximum tolerable specimen thicknesses for diffraction contrast imaging of defects by low-keV STEM, high-keV STEM and TEM and comparison of the width of dislocation line contrast.

\section{Additional file}

Additional file 1. Additional information for defects analysis by STEM in a SEM.

\section{Abbreviations}

ADF: annular dark field; BF: bright field; CCD camera: charge-coupled device camera; DF: dark field; FIB: focused-ion beam; low-keV STEM: STEM in a scanning electron microscope; SEM: scanning electron microscopy/microscope; STEM: scanning transmission electron microscopy; TED: transmission electron diffraction; TEM: transmission electron microscopy; TLD: through-the-lens detector; ZB: undiffracted zero-order beam.

\section{Authors' contributions}

CS, EM and DG conceived the experiments. CS performed the S(T)EM experiments. EM helped with FIB-based sample preparation. MM significantly contributed to the interpretation of the results. CS and DG wrote the manuscript. All authors read and approved the final manuscript.

\section{Acknowledgements}

We gratefully acknowledge funding by the Deutsche Forschungsgemeinschaft and Open Access Publishing Fund of Karlsruhe Institute of Technology.

\section{Competing interests}

The authors declare that they have no competing interests.

Availability of data and materials

All the research data in this study can be provided by the corresponding author upon reasonable request. 


\section{Funding}

This work was supported by the Deutsche Forschungsgemeinschaft (DFG) under Contract Number GE 841/20-2.

\section{Publisher's Note}

Springer Nature remains neutral with regard to jurisdictional claims in published maps and institutional affiliations.

Received: 3 December 2018 Accepted: 2 March 2019

Published online: 09 March 2019

\section{References}

1. Hirsch, P., Howie, A., Nicholson, R.B., Pashley, D.W., Whelan, M.J.: Electron microscopy of thin crystals, 2nd edn, pp. 247-275. Springer, New York (1977)

2. Williams, D.B., Carter, C.B.: Transmission electron microscopy: a textbook for materials science, 2nd edn, pp. 441-459. Springer, New York (2009)

3. Thomas, G., Goringe, M.J.: Transmission electron microscopy of materials, pp. 143-169. Wiley, New York (1979)

4. Nikolaichick, V.I., Khodos, I.I.: A review of the determination of dislocation parameters using strong- and weak-beam electron microscopy. J. Microsc. 155, 123-167 (1989)

5. Cowley, J.M.: Image contrast in a transmission scanning electron microscope. Appl. Phys. Lett. 15, 58 (1969)

6. Maher, D.M., Joy, D.C.: The formation and interpretation of defect images from crystalline materials in a scanning transmission electron microscope. Ultramicroscopy 1, 239-253 (1976)

7. Humphreys, C.J.: Fundamental concepts of STEM imaging. Ultramicroscopy 7,7-12 (1981)

8. Perovic, D.D., Howie, A., Rossouw, C.J.: On the image contrast from dislocations in high-angle annular dark-field scanning transmission electron microscopy. Phil. Mag. Lett. 67, 261-272 (1993)

9. Phillips, P.J., Brandes, M.C., Mills, M.J., De Graef, M.: Diffraction contrast STEM of dislocations: imaging and simulations. Ultramicroscopy 111, 1483-1487 (2011)

10. Su, X.J., Niu, M.T., Zeng, X.H., Huang, J., Zhang, J.C., Zhang, J.P., Wang, J.F., Xu, K.: Identifying dislocations and stacking faults in GaN films by scanning transmission electron microscopy. Mater. Res. Express. 3, 086401 (2016)

11. Zhu, Y., Ophus, C., Toloczko, M.B., Edwards, D.J.: Towards bend-contourfree dislocation imaging via diffraction contrast STEM. Ultramicroscopy 193, 12-23 (2018)

12. Sun, C., Muller, E., Meffert, M., Gerthsen, D.: On the progress of scanning transmission electron microscopy (STEM) imaging in a scanning electron microscope. Microsc. Microanal. 24, 99-106 (2018)

13. Callahan, P.G., Stinville, J.C., Yao, E.R., Echlin, M.P., Titus, M.S., de Graef, M., Gianola, D.S., Pollock, T.M.: Transmission scanning electron microscopy: defect observations and image simulations. Ultramicroscopy. 186, 49-61 (2018)

14. Langford, R.M., Rogers, M.: In situ lift-out: steps to improve yield and a comparison with other FIB TEM sample preparation techniques. Micron. 39, 1325-1330 (2008)

15. Lyman, C.E., Newbury, D.E., Goldstein, J.I., Williams, D.B., Romig Jr, A.D. Armstrong, J.T., Echlin, P., Fiori, C.E., Joy, D.C., Lifshin, E., Peters, K.R.: Scanning electron microscopy, $\mathrm{X}$-ray microanalysis, and analytical electron microscopy: a laboratory workbook, pp. 11-13. Plenum Press, New York (1990)

16. Jain, S.C., Willander, M., Narayan, J., Overstraeten, R.V.: III-nitrides: growth, characterization, and properties. J. Appl. Phys. 87, 965 (2000)

17. Ponce, F.A., Cherns, D., Young, W.T., Steeds, J.W.: Characterization of dislocations in GaN by transmission electron diffraction and microscopy techniques. Appl. Phys. Lett. 69, 770 (1996)

18. Hull, D., Bacon, D.J.: Introduction to dislocations, 5th edn, pp. 109-136. Elsevier, Oxford (2011)

19. Wu, X.H., Brown, L.M., Kapolnek, D., Keller, S., Keller, B., DenBaars, S.P., Speck, J.S.: Defect structure of metal-organic chemical vapor depositiongrown epitaxial (0001) GaN/Al2O3. J. Appl. Phys. 80, 3228 (1996)

20. Hirsch, P.B., Lozano, J.G., Rhode, S., Horton, M.K., Moram, M.A., Zhang, S. Kappers, M.J., Humphreys, C.J., Yasuhara, A., Okunishi, E., Nellist, P.D.:The dissociation of the $[\mathrm{a}+\mathrm{c}]$ dislocation in GaN. Phil. Mag. 93, 3925-3938 (2013)

21. Cockayne, D.J.H., Ray, I.L.F., Whelan, M.J.: Investigations of dislocation strain fields using weak beams. Phil. Mag. 20, 1265-1270 (1969)

22. Potin, V., Ruterana, P., Nouet, G.: HREM study of stacking faults in GaN layers grown over sapphire substrate. J. Phys.: Condens. Matter 12, 10301-10306 (2000)

\section{Submit your manuscript to a SpringerOpen ${ }^{\circ}$ journal and benefit from:}

- Convenient online submission

- Rigorous peer review

- Open access: articles freely available online

- High visibility within the field

- Retaining the copyright to your article

Submit your next manuscript at $\boldsymbol{\nabla}$ springeropen.com 